\title{
PRODUCTS OF $k^{\prime}$-SPACES
}

\author{
R. W. BAGLEY AND D. D. WEDDINGTON
}

We call a topological space $X$ a $k^{\prime}$-space if $A \subset X, x \in \bar{A}$ implies the existence of a compact subset $K$ of $X$ such that $x \in \mathrm{Cl}(A \cap K)$ (where $\mathrm{Cl}$ stands for closure) [1]. A characterization of $k^{\prime}$-spaces is given in [8]. E. A. Michael has shown that a Hausdorff space is locally compact if its product with every $k$-space is a $k$-space [6]. We obtain an analogous result for $k^{\prime}$-spaces which implies the existence of $k$-spaces which are not $k^{\prime}$-spaces. It is stated in [1] that such spaces exist as opposed to remarks in [4] and [7]. We show that a $T_{1}$ space $X$ is discrete if $X \times Y$ is a $k^{\prime}$-space for every $k^{\prime}$-space $Y$. Thus a nontrivial product theorem for $k^{\prime}$-spaces must involve additional conditions on both factors, in contrast to Cohen's Theorem [3] $(X \times Y$ is a $k$-space for $X$ locally compact and $Y$ a $k$-space-see for instance [2]). We do show that $X \times Y$ is a $k^{\prime}$-space if both $X$ and $Y$ are $T_{1}, k^{\prime}$-spaces and $X \times Y$ has a nested neighborhood base at each of its points. Also the product of two $T_{1}$ spaces with a nested neighborhood base at each point is a $k$-space if one of the spaces is a $k^{\prime}$-space and the other is a $k$-space.

Theorem 1. If $X$ is a nondiscrete $T_{1}$ space, then there is a $k^{\prime}$-space $Y$ such that $X \times Y$ is not a $k^{\prime}$-space.

Proof. Let $\left\{x_{\alpha}: \alpha \in D\right\}$ be a net converging to $x$ such that $x_{\alpha} \neq x$, $\alpha \in D$. Let $Y_{1}=\{(\alpha, n): \alpha \in D$ and $n=1,2,3, \cdots\}$ and let $Y=Y_{1}$ $\cup\{z\}$. The topology on $Y$ is as follows: $Y_{1}$ is discrete and the open sets containing $z$ contain all but a finite number of elements of each set $A=\{(\alpha, n): n=1,2,3, \cdots\}$ for $\alpha \in D$. It is easy to see that $Y$ is a $k^{\prime}$-space since each compact subset intersects only finitely many of the sets $A$. On the other hand $(x, z)$ is a limit point of the set $C=\left\{\left(x_{\alpha},(\alpha, n)\right): \alpha \in D, n=1,2,3, \cdots\right\}$ but clearly not a limit point of $C \cap K$ for any compact set $K$. Thus $X \times Y$ is not a $k^{\prime}$-space.

REMARK. If $X$ is a nondiscrete locally compact $T_{1}$ space, then there is a $k^{\prime}$-space $Y$ (as in the proof of the theorem) such that $X \times Y$ is a $k$-space which is not a $k^{\prime}$-space. The space $Y$ is paracompact. As a matter of fact every open cover has a discrete open refinement. Also $Y$ can be slightly modified to make it a $\mathrm{CW}$-complex.

Before proving Theorem 2 we need a lemma on product spaces with nested neighborhood bases. From this point on we assume that $X$ and $Y$ are $T_{1}$ spaces.

Received by the editors August 14, 1968. 
Lemma. If $X \times Y$ has a nested neighborhood base at $(x, y) \in \bar{A}-A$ and there are neighborhoods $U$ of $x$ and $V$ of $y$ such that $\{x\} \times V \cap A=\varnothing$ and $U \times\{y\} \cap A=\varnothing$, then there is a net $\left\{\left(x_{\alpha}, y_{\alpha}\right): \alpha \in D\right\}$ in $A$ which converges to $(x, y)$ and, for each $\alpha_{0} \in D$, there are neighborhoods $R$ of $x$ and $S$ of $y$ such that $x_{\alpha} \notin R, y_{\alpha} \notin S$ for $\alpha<\alpha_{0}$.

Proof. We can choose a nested neighborhood base at $(x, y)$ of the form $\left\{U_{\alpha} \times V_{\alpha}: \alpha \in D\right\}$ where $D$ is directed as follows: $\alpha<\beta$ iff $U_{\alpha} \times V_{\alpha} \supset U_{\beta} \times V_{\beta}$. We well order the set $B=\left\{U_{\alpha} \times V_{\alpha}: \alpha \in D\right\}$ by $\prec$ and for each $\alpha \in D$ choose the first element $U_{\beta} \times V_{\beta}$ of $B$ such that $\left(U_{\alpha}-U_{\beta} \times V_{\alpha}-V_{\beta}\right) \cap A \neq \varnothing$. If for some fixed $\alpha$ no such choice is possible, then $A \cap\left(\cup\left\{U_{\alpha}-U_{\beta} \times V_{\alpha}-V_{\beta}: \beta \in D\right\}\right)=\varnothing$. Since $B$ is nested, $U\left\{U_{\alpha}-U_{\beta} \times V_{\alpha}-V_{\beta}\right\}=U_{\alpha}-\{x\} \times V_{\alpha}-\{y\}$. We can assume that $U_{\alpha} \times V_{\alpha} \subset U \times V$ where $U$ and $V$ are the neighborhoods given in the hypothesis. Thus, $\varnothing=A \cap\left(U_{\alpha}-\{x\} \times V_{\alpha}-\{y\} \cup U_{\alpha}\right.$ $\left.\times\{y\} \cup\{x\} \times V_{\alpha}\right) \supset A \cap\left(U_{\alpha} \times V_{\alpha}-\{(x, y)\}\right)$. This contradicts the fact that $(x, y) \in \bar{A}-A$. Now, for each $\alpha \in D$, we take $\left(x_{\alpha}, y_{\alpha}\right)$ $\in\left(U_{\alpha}-U_{\beta} \times V_{\alpha}-V_{\beta}\right) \cap A$ where $U_{\beta} \times V_{\beta}$ is chosen as indicated above. Let $\alpha_{0} \in D$ and let $R=U_{\beta_{0}}, S=V_{\beta_{0}}$ again choosing $\beta_{0}$ such that $U_{\beta_{0}} \times V_{\beta_{0}}$ is the first element of $Q$ satisfying $\left(U_{\alpha_{0}}-U_{\beta_{0}}\right.$ $\left.\times V_{\alpha_{0}}-V_{\beta_{0}}\right) \cap A \neq \varnothing$. We complete the proof by showing that $x_{\alpha} \notin U_{\beta_{0}}$ and $y_{\alpha} \notin V_{\beta_{0}}$ for $\alpha<\alpha_{0}$. Suppose there is $\alpha<\alpha_{0}$ such that $x_{\alpha} \in U_{\beta_{0}}$ or $y_{\alpha} \in V_{\beta_{0}}$. Since $U_{\beta_{0}} \times V_{\beta_{0}} \subset U_{\alpha_{0}} \times V_{\alpha_{0}} \subset U_{\alpha} \times V_{\alpha},\left(U_{\alpha}-U_{\beta_{0}}\right.$ $\left.\times V_{\alpha}-V_{\beta_{0}}\right) \cap A \neq \varnothing$. It follows that $U_{\beta} \times V_{\beta} \prec U_{\beta_{0}} \times V_{\beta_{0}}$. Since $x_{\alpha} \notin U_{\beta}$ and $y_{\alpha} \notin V_{\beta}$, we have $U_{\beta} \times V_{\beta} \subset U_{\alpha_{0}} \times V_{\alpha_{0}}$. This implies that $U_{\beta_{0}} \times V_{\beta_{0}} \prec U_{\beta} \times V_{\beta}$ which is a contradiction. This completes the proof.

By a routine use of the definition of subnet [5] we can establish the fact that any net in $\left\{x_{\alpha}\right\}$ (resp. $\left\{y_{\alpha}\right\}$ ) which converges to $x$ (resp. $y$ ) is a subnet of $\left\{x_{\alpha}\right\}$ (resp. $\left\{y_{\alpha}\right\}$ ). We use this fact in the proof of Theorem 2. We also use the following characterization of $k$-spaces established in [8]: A topological space $X$ is a $k$-space iff, for each subset $A$ and $x \in \bar{A}$, there is a closed $k$-subspace $C$ such that $x \in \mathrm{Cl}(A \cap C)$.

Theorem 2. If $X$ is a $k^{\prime}$-space and $Y$ is a $k^{\prime}$-space ( $k$-space) and $X \times Y$ has a nested neighborhood base at each point, then $X \times Y$ is a $k^{\prime}$-space (k-space).

Proof. Let $A$ be a subset of $X \times Y$ and let $x \in \bar{A}-A$. If the neighborhoods $U$ and $V$ of the lemma do not exist, then our conclusion follows routinely. Thus there is a net $\left\{\left(x_{\alpha}, y_{\alpha}\right)\right\}$ in $A$ converging to $(x, y)$ and satisfying the conclusion of the lemma. Since $X$ is a 
$k^{\prime}$-space, there is a compact subset $K$ of $X$ such that $x \in \mathrm{Cl}\left(\left\{x_{\alpha}\right\} \cap K\right)$. Thus there is a net $\left\{x_{\gamma}\right\}$ in $\left\{x_{\alpha}\right\} \cap K$ which converges to $x$. By the note which follows the proof of the lemma $\left\{x_{\gamma}\right\}$ is a subnet of $\left\{x_{\alpha}\right\}$ and $\left\{y_{\gamma}\right\}$ converges to $y$, being a subnet of $\left\{y_{\alpha}\right\}$. Since $Y$ is a $k$-space, there is a closed $k$-subspace $C$ of $Y$ (in case $Y$ is a $k^{\prime}$-space $C$ can be chosen compact) such that $y \in \mathrm{Cl}\left(\left\{y_{\gamma}\right\} \cap C\right)$. Finally we obtain a subnet of $\left\{\left(x_{\alpha}, y_{\alpha}\right)\right\}$ in $K \times C \cap A$. Thus $(x, y) \in \mathrm{Cl}(K \times C \cap A)$. If $C$ is a $k$-space, then $K \times C$ is a $k$-space [2] and if $C$ is compact $K \times C$ is also. Thus, in case $Y$ is a $k$-space, $X \times Y$ is a $k$-space and a $k^{\prime}$-space when $Y$ is.

\section{BibliograPHY}

1. A. V. Arhangel'skil, Bicompact sets and the topology of spaces, Soviet Math. Dokl. 4 (1963), 561-564.

2. R. W. Bagley and J. S. Yang, On k-spaces and function spaces, Proc. Amer. Math. Soc. 17 (1966), 703-705.

3. D. E. Cohen, Spaces with weak topology, Quart. J. Math. Oxford Ser. 5 (1954), 77-80.

4. Z. Frolík, Topologically complete spaces (summary of author's results), Comment. Math. Univ. Carolinae 8 (1960), 3-15.

5. J. L. Kelley, General topology, Van Nostrand, New York, 1955.

6. E. Michael, On the product of k-spaces, Notices Amer. Math. Soc. 14 (1967), 688.

7. H. Tamano, $A$ note on the pseudo-compactness of the product of two spaces, Mem. Coll. Sci. Univ. Kyoto Ser. A Math. 33 (1960), 225-230.

8. D. Weddington, $k$-spaces, Doctoral Dissertation, University of Miami, Coral Gables, Fla., 1968.

UNIVERSITY OF MiaMI 